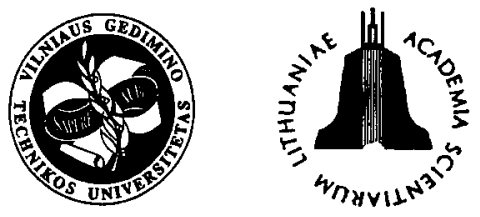

\title{
SHAKEDOWN LOADING OPTIMIZATION UNDER CONSTRAINED RESIDUAL DISPLACEMENTS - FORMULATION AND SOLUTION FOR CIRCULAR PLATES
}

\author{
Ela Jarmolajeva ${ }^{1}$, Juozas Atkočiūnas ${ }^{2}$ \\ Dept of Structural Mechanics, Vilnius Gediminas Technical University, \\ Sauletekio al. 11, LT-2040 Vilnius, Lithuania
}

Received 15 Sept 2001; accepted 10 Dec 2001

\begin{abstract}
The adapted plate load optimization problem is formulated applying the non-linear mathematical programming methods. The load variation bounds satisfying the optimality criterion in concert with the strength and stiffness requirements are to be identified. The stiffness constraints are realized via residual displacements. The dual mathematical programming problems cannot be applied directly when determining actual stress and strain fields of plate: the strained state depends upon the loading history. Thus the load optimization problem at shakedown is to be stated as a couple of problems solved in parallel: the shakedown state analysis problem and the verification of residual deflections bounds. The Rozen project gradient method is applied to solve the cyclically loaded non-linear shakedown plate stress and strain evaluation and that of the load optimization problems. The mechanical interpretation of Rozen optimality criterions allows to simplify the shakedown plate optimization mathematical model and solution algorithm formulations.
\end{abstract}

Keywords: shakedown, loading optimization problem, mathematical models of circular plates, mathematical programming theory.

\section{Introduction}

The present paper continues the investigations of mathematical programming method applications for perfectly elastic-plastic structures in the range of the shakedown theory [1-12]. The Rozen project gradient method [13] is applied to solve the cyclically loaded non-linear shakedown plate stress and strain evaluation and that of the load optimization problems. The mechanical interpretation of the Rozen algorithm optimality criterions in the structural analysis problem was explained in the investigations $[14,15]$ based on the application of the known in the mathematical programming method KuhnTucker conditions. The Kuhn-Tucker conditions mean the elastic or elastic-plastic structure compatibility equations (note that for the current moment such an interpretation was not met in scientific publications; these equations were applied only to confirm the solution to be the global one [12]). The mechanical interpretation of Rozen optimality criterions allows to simplify the shakedown plate optimization mathematical model and solution algorithm formulations. The applications related to the circular bendable plate described by the Mises yield conditions are analysed in the paper.

\footnotetext{
I E-mail: Ela.Chraptovic@st.vtu.It

2 E-mail: Juozas.Atkociunas@st.vtu.lt
}

A perfectly elastic-plastic plate material, geometry, load application points are prescribed. The quasistatic variable load is described by the time independent upper and lower bounds of variation. The detailed loading history is not under consideration, as the loading is sloped by the above-mentioned variation bounds, being the unknown variables in the problem to be considered. The plate load optimization problem is stated as follows: find the load variation bounds which satisfy a prescribed optimality criterion, strength and stiffness constraints. The dissipative structure stress and strain fields depend on a certain unique loading history, therefore to define the unique plate deflections for the given load variation bounds is rather difficult or even impossible. Thus, an indirect evaluation of all the expected plastic deformation trajectories seems to be the only possibility to solve the real problem [7, 16-22]. Consequently, the load optimization problem couples two structural mechanics problems. The first problem tasks include an evaluation of the statically admissible residual plate bending moments corresponding to the complementary energy minimum magnitude. The first problem solution influences the solution process of the second problem, to evaluate the residual displacements bounds as variables being included into the stiffness constraints. The Rozen criterion mathematical-mechanical interpretation is a connecting value for the above-mentioned first and second problems. 
The investigation material in the paper is presented in the following order. The plate discretization by finite elements is analysed in the second section, where the plate discrete model main equations and relationships are characterised in brief. These equations and relationships are applied in the third section when formulating the mathematical model aimed at evaluating the residual displacements bounds. The problem optimal solution Kuhn-Tucker conditions together with those of mathematical-mechanical interpretations are given in the fourth section. The discrete adapted plate load optimization problem formulations and obtained formulations analysis are described in the fifth section. The proposed problem solution algorithm is presented in the sixth section; the seventh section is assigned to numerical applications.

\section{Plate discrete model main equations and relation- ships}

The circular plate geometry, sandwich cross-section dimensions and material are prescribed. A plate discretized by means of the equilibrium finite elements $[23,24]$. An element $k(k=1,2, \ldots, n ; k \in K)$ contains three nodal points $l=1,2,3(l \in L)$. Hence, the vector of bending moments due to the applied cylindrical coordinate system is: $\mathbf{M}_{k}=\left(M_{\rho, k 1}, M_{\theta, k 1}, M_{\rho, k 2}\right.$, $\left.M_{\theta, k 2}, M_{\rho, k 3}, M_{\theta, k 3}\right)^{T}=\left(\mathbf{M}_{k 1}, \mathbf{M}_{k 2}, \mathbf{M}_{k 3}\right)^{T}$. Here $\mathbf{M}_{k l}=\left(M_{\rho, k l}, M_{\theta, k l}\right)^{T}$. The indices $\rho$ and $\theta$ define a certain type of bending moments with positive directions as shown in Fig 1. The bending moments approximation functions read:

$$
\mathbf{M}_{k}(\rho)=\left[\mathbf{N}_{k}(\rho)\right] \mathbf{M}_{k} .
$$

Here $\left[\mathbf{N}_{k}(\rho)\right]$ is $k$-th element internal forces approximation matrix.

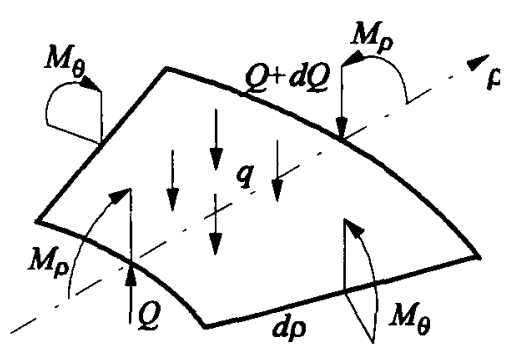

Fig 1. Plate element

The functions (1) do not identically satisfy the plate element equilibrium equation

$$
\begin{gathered}
\frac{d^{2}}{d r^{2}}\left(r M_{\rho}\right)-\frac{d}{d r} M_{\theta}+r q(\rho)=0 \text { or } \\
{[\propto \mathcal{M}] \mathbf{M}(\rho)=q(\rho) .}
\end{gathered}
$$

Therefore the discrete model equilibrium is ensured for the plate elements and main nodes. The substitution of the relationship (1) by the formula (2) after differentiation results in the following algebraic finite element equi- librium equation:

$$
\left[A_{k}(\rho)\right] \mathbf{M}_{k}=q_{k}(\rho) \text {. }
$$

Here $\left[A_{k}(\rho)\right]=[\Theta A]\left[\mathbf{N}_{k}(\rho)\right]$. For a uniformly distributed load case, $q_{k}(\rho)=q_{k}$; the latter case is to be analysed in the paper. The separate elements are coupled with the system in order to ensure the bending moments $M_{\rho}$ and shearing forces $Q$ continuity, when formulating the equilibrium equations for the first and second main nodes of separate elements. Taking into account boundary conditions the plate $m$ dimension equilibrium equations system reads:

$$
\sum_{k}\left[A_{k}\right] \mathbf{M}_{k}=\mathbf{F} \text { or }[A] \mathbf{M}=\mathbf{F} .
$$

The plate geometrical equations are formulated applying the stress virtual increment principle:

$$
\delta \mathbf{F}^{T} \mathbf{u}=\sum_{k} \int_{A_{k}} \delta \mathbf{M}_{k}^{T}(\rho)[\mathscr{D}] \mathbf{M}_{k}(\rho) d A .
$$

An application of the relationship $\mathbf{M}_{k}(\rho)=\left[\mathbf{N}_{k}(\rho)\right] \mathbf{M}_{k}$ and that of the equations $\sum_{k}\left[A_{k}\right] \mathbf{M}_{k}=\mathbf{F}$ result in the equality:

$$
\sum_{k} \boldsymbol{\delta} \mathbf{M}_{k}^{T}\left[A_{k}\right]^{T} \mathbf{u}=\sum_{k} \delta \mathbf{M}_{k}^{T}\left[D_{k}\right]^{T} \mathbf{M}_{k}
$$

Here $k$-th element flexibility matrix $\left[D_{k}\right]$ is obtained applying the formula

$$
\left[D_{k}\right]=\int_{A_{k}}\left[\mathbf{N}_{k}(\rho)\right]^{T}[\mathscr{D}]\left[\mathbf{N}_{k}(\rho)\right] d A
$$

Following the above-mentioned operations, the geometrical equations for the separate element are obtained:

$$
\left[A_{k}\right]^{T} \mathbf{u}-\left[D_{k}\right] \mathbf{M}_{k}=\mathbf{0}, \quad k \in K ;
$$

when coupled with the whole plate discrete model, read

$$
[A]^{T} \mathbf{u}-[D] \mathbf{M}=\mathbf{0} .
$$

Here $[D]$ is the quasi-diagonal plate elements flexibility matrix [24]. The physical meaning of the displacement vector components is defined by the order resulted from the dual relationship with the equilibrium equations $[A] \mathbf{M}=\mathbf{F}$. An equilibrium finite elements were applied in investigation [25] when analysing the elastic-plastic plate subjected by monotonically increasing loading, in case of linear yield conditions.

The plate transition to the plasticity stage is defined by the non-linear Mises yield condition $M_{\rho}^{2}-M_{\rho} M_{\theta}+M_{\theta}^{2}=M_{0}^{2}$. The plate limit bending moment $M_{0 k}$ is prescribed and constant per finite element area, ie $M_{0 k}=$ const . The Mises yield condition is verified for each finite element node:

$$
\varphi_{k l}=\left(M_{0 k}\right)^{2}-\mathbf{M}_{k l}^{T}[\Phi] \mathbf{M}_{k l} \geq 0, k \in K, l \in L
$$

Here $[\Phi]$ is the Mises yield condition coefficient matrix. 
When analyzing the perfectly elastic-plastic plate subjected to cyclic load $\mathbf{F}(t)$, it makes sense to describe its stress field by means of the elastic $\mathbf{M}_{e}$ and that of the residual $\mathbf{M}_{r}$ bending moments. Applying the known formula of structural mechanics for elastic design bending moments vs external load, $\mathbf{F}$ relationship reads:

$$
\mathbf{M}_{e}=[\alpha] \mathbf{F} .
$$

Actually, in engineering practice an elastic internal force influence matrix [a] for elastic internal force evaluation is applied rarely. When the problem mathematical model is formulating, the internal forces influence matrix $[a]$ expressively illustrates an interaction of the internal forces $\mathbf{M}_{e}$ and load F. Due to methodical aspect of the above-mentioned the matrix $[a]$ is applied extensively in the paper. Let us say that the actual load process is described via the time $t$ independent of load variation bounds $\mathbf{F}_{\text {sup }}$ and $\mathbf{F}_{\text {inf }}\left(\mathbf{F}_{\text {inf }} \leq \mathbf{F}(t) \leq \mathbf{F}_{\text {sup }}\right)$. Then the certain bending moments distribution $\mathbf{M}_{e j}$ is calculated for each external forces combination $j$ (ie for the vector of loads variation bounds components), the all combinations being coupled with the set $j=1,2, \ldots, p, j \in J$. These vectors represent the polyhedron apexes of the loading process $\mathbf{M}_{e}(t)=[\alpha] \mathbf{F}(t)$ variation field. The total number of apexes makes the set $j \in J$. The extreme bending moments $\mathbf{M}_{e j}$ are linear functions of the load variation bounds $\mathbf{F}_{\text {inf }}, \mathbf{F}_{\text {sup }}$ (11). The Mises yield conditions then read:

$$
\begin{aligned}
& \left(M_{e \rho, j}+M_{r \rho}\right)^{2}-\left(M_{e \rho, j}+M_{r \rho}\right)\left(M_{e \theta, j}+M_{r \theta}\right)+ \\
& +\left(M_{e \theta, j}+M_{r \theta}\right)^{2} \leq\left(M_{0}\right)^{2} .
\end{aligned}
$$

The statically admissible bending moments $\mathbf{M}_{r}$ satisfy the equilibrium equations $[A] \mathbf{M}_{r}=0$, ie they are self-balanced. The $k$-th element $j$-th node yield condition reads:

$$
\begin{aligned}
& \left(M_{e \rho, k l, j}+M_{r \rho, k l}\right)^{2}-\left(M_{e \rho, k l, j}+M_{r \rho, k l}\right)\left(M_{e \theta, k l, j}+\right. \\
& \left.+M_{r \theta, k l}\right)+\left(M_{e \theta, k l, j}+M_{r \theta, k l}\right)^{2} \leq\left(M_{0 k}\right)^{2}
\end{aligned}
$$

or

$$
\begin{gathered}
\varphi_{k l, j}=\left(M_{0 k}\right)^{2}-\mathbf{M}_{k l, j}^{T}[\Phi] \mathbf{M}_{k l, j} \geq 0, \\
\mathbf{M}_{k l, j}=\mathbf{M}_{e k l, j}+\mathbf{M}_{r k l}, k \in K, l \in L, j \in J .
\end{gathered}
$$

The geometrical equations (9) read:

$$
[A]^{T} \mathbf{u}_{r}=[D] \mathbf{M}_{r}+\boldsymbol{\Theta}_{p} .
$$

Here $\mathbf{u}_{r}$ is the vector of residual displacements; $\Theta_{p}=\left(\Theta_{p k l}\right)^{T}$ is the vector of plastic strains. The vector $\Theta_{p}$ is calculated applying the formula:

$$
\Theta_{p k l}=2 \sum_{j} \lambda_{k l, j}[\Phi] \mathbf{M}_{k l, j}, \quad k \in K, \quad l \in L, \quad j \in J
$$

Note that adapted plate load optimization problem mathematical models and numerical experiments in the current investigation are provided for linear Kirchhoff plate.

\section{Plate analysis mathematical model}

An elastic plate behaviour can be qualitatively represented after a qualified analysis of the plate load vs deflection is provided. The plate behaves elastically if the distributed load intensity parameter $q$ does not exceed the limiting magnitude $q_{T}$. Then the relationship load versus deflection is linear. In case when $q>q_{T}$, the plastic strains leading to the plate stiffness degradation appear. Then the relationship load vs deflection is non-linear. After a certain load parameter magnitude $q_{0}$ is achieved, the plate collapses plastically (in case of the perfectly elastic-plastic material) or its deflections develop very fast in case of the elastic-plastic hardening material. The perfectly elastic-plastic plate behaviour analysis is under consideration in the present investigation, thus the limit plate state is described by the load parameter magnitude $q_{0}$. The identification of this magnitude in the structural analysis is known as the limit equilibrium problem.

The actual stress and strain field evaluation of the plastically deformed plate being in the state prior to the plastic collapse is under request of usual structural engineering practice. The plate subjected to the variable load $\mathbf{F}_{\text {inf }} \leq \mathbf{F}(t) \leq \mathbf{F}_{\text {sup }}$ at the state prior to the plastic collapse is the object of shakedown theory investigation. The residual bending moments, deflections and strains evaluation is the aim of usual shakedown theory investigation. The above-mentioned values are determined by solving the plate analysis problem for prescribed load variation bounds $\mathbf{F}_{\text {sup }}$ and $\mathbf{F}_{\text {inf }}$.

Let us consider the bendable shakedown plate state analysis problem in detail. By solving the shakedown plate analysis problem in static formulation the residual bending moments $\mathbf{M}_{r}$ are to be determined. The problem static formulation is due to the minimum complementary energy principle, reading $[2,4,5]$ :

of all statically possible residual bending moments of plate at shakedown is the minimum complementary energy corresponding one.

The problem due to the above-mentioned principle reads:

find

$$
\min \frac{1}{2} \sum_{k} \mathbf{M}_{r k}^{T}\left[D_{k}\right] \mathbf{M}_{r k},
$$

subject to

$$
\begin{gathered}
\sum_{k}\left[A_{k}\right] \mathbf{M}_{r k}=\mathbf{0} \\
\varphi_{k l, j}=C_{k}-\left(\mathbf{M}_{e k l, j}+\mathbf{M}_{r k l}\right)^{T}[\Phi]\left(\mathbf{M}_{e k l, j}+\mathbf{M}_{r k l}\right) \geq 0 \\
C_{k}=\left(M_{0 k}\right)^{2}, \quad k \in K, \quad l \in L, \quad j \in J .
\end{gathered}
$$


The analysis problem mathematical model (15)-(17) yield conditions

$$
\varphi_{k l, j}=C_{k}-\left(\mathbf{M}_{e k l, j}+\mathbf{M}_{r k l}\right)^{T}[\Phi]\left(\mathbf{M}_{e k l, j}+\mathbf{M}_{r k l}\right) \geq 0
$$

take into account all polyhedron apexes $j \in J$ of elastic bending moments distribution field $\mathbf{M}_{e}(t)=[\alpha] \mathbf{F}(t)$. Therefore the analysis problem is solved for the prescribed limit bending moments $\mathbf{M}_{0}=\left(M_{01}, M_{02}, \ldots, M_{0 n}\right)^{T}$ and that of the elastic bending moments $\mathbf{M}_{e j}$. The plate shakedown state bending moments vector $\mathbf{M}_{r}$ is the unknown value of the non-linear convex mathematical programming problem (15)(17). The problem (15)-(17) optimal solution is denoted as $\mathbf{M}_{r}^{*}$. The shakedown of the plate is ensured by the existence of the statically admissible residual bending moments vector $\mathbf{M}_{r}$ in contradiction to the minimum complementary energy magnitude $[7,17,20-22]$. The plate shakedown state residual deflections and curvatures are identified by solving the dual of the (15)-(17) problem. The complementarity conditions are incorporated in this problem. These conditions do not allow to evaluate directly an unloading phenomenon (one can meet it when for an actual loading process $\lambda_{i}>0$ and $\varphi_{i}>0 ; i \in I$ ). An objective function magnitude $\frac{1}{2} \sum_{k} \mathbf{M}_{r k}^{T}\left[D_{k}\right] \mathbf{M}_{r k}$ at unloading can numerically exceed an optimal magnitude $\frac{1}{2} \sum_{k} \mathbf{M}_{r k}^{* T}\left[D_{k}\right] \mathbf{M}_{r k}^{*}$. Consequently, it is impossible to define uniquely the plate residual deflections. In this case the problem (15)-(17) and its dual one are to be solved iteratively following a certain loading history.

The mathematical model (15)-(17) can be rewritten simpler. The residual bending moments $\mathbf{M}_{r}=\left(\mathbf{M}_{r}^{\prime}, \mathbf{M}_{r}^{\prime \prime}\right)^{T}$ are expressed by means of the force method variables $\mathbf{x}$ (bending moments $\mathbf{M}_{r}$ ) applying the known Jordan transformations:

$$
\mathbf{M}_{r}=[B]^{T} \mathbf{x} .
$$

The matrix $[B]=\left[-\left[A^{\prime \prime}\right]^{T}\left(\left[A^{\prime}\right]^{T}\right)^{-1},[\mathrm{I}]\right]$ contains submatrices $\left[A^{\prime}\right],\left[A^{\prime \prime}\right]$ being the compound submatrices of the matrix $[A]$, (see $[B]^{T}=\left[\begin{array}{c}-\left[A^{\prime}\right]^{-1}\left[A^{\prime \prime}\right] \\ {[I]}\end{array}\right]$ ). The matrix allows the shakedown circular plate analysis problem (15) $-(17)$ to be transformed as follows:

find

$$
\begin{gathered}
\min \frac{1}{2} \sum_{k} \mathbf{M}_{r}^{" T}[B]\left[D_{k}\right][B]^{T} \mathbf{M}_{r}^{\prime \prime}= \\
=\min \frac{1}{2} \sum_{k} \mathbf{M}_{r}^{" T}[\tilde{D}] \mathbf{M}_{r}^{\prime \prime},
\end{gathered}
$$

subject to

$$
\begin{gathered}
\varphi_{k l, j}=C_{k}-\left(\mathbf{M}_{e k l, j}+\left[B_{k l}\right]^{T} \mathbf{M}_{r}^{\prime \prime}\right)^{T}[\Phi]\left(\mathbf{M}_{e k l, j}+\right. \\
\left.+\left[B_{k l}\right]^{T} \mathbf{M}_{r}^{\prime \prime}\right) \geq 0, \quad C_{k}=\left(M_{0 k}\right)^{2}, \\
k \in K, \quad l \in L, \quad j \in J .
\end{gathered}
$$

The residual bending moments $\mathbf{M}_{r}^{\prime \prime}$ are the unknowns of the problem (19)-(20).

\section{Kuhn-Tucker conditions and plate analysis prob- lem}

The cyclically loaded elastic-plastic plate residual stresses evaluation problem (19)-(20) in terms of mathematical programming theory can be written as follows:

find

$$
\min \left\{\mathscr{F}(\mathbf{x})=\frac{1}{2} \mathbf{x}^{T}[\tilde{D}] \mathbf{x} \mid \mathbf{x} \in \mathscr{L}\right\} .
$$

Here $\mathscr{Z}=\left\{\mathbf{x} \mid \varphi_{i}(\mathbf{x}) \geq 0\right.$ for $\left.i=1,2, \ldots, \zeta\right\}$ is an admissible set of variables $\mathbf{x}$ (here equalities $h_{i}(\mathbf{x})=0$ are eliminated). The functions $\varphi_{i}(\mathbf{x}) \geq 0$ are convex, the matrix $[\widetilde{D}]$ is positively defined. The global solution $\mathbf{x}^{*} \in \mathscr{L}$ minimizes an objective function $\mathscr{F}\left(\mathbf{x}^{*}\right)$. The Rozen project gradient method [13] is known as an algorithm for convex mathematical programming problem solution. Here the objective function gradient $\nabla \mathscr{F}(\mathbf{x})$ is projected onto the admissible set $\mathscr{L}$ edge. The vector $\mathbf{x}^{*}$ is an optimal solution if it satisfies the Rozen algorithm optimality criterion:

$$
\begin{gathered}
\left\{[I]-\left[\nabla^{T} \varphi\left(\mathbf{x}^{*}\right)\right]\left(\left[\nabla \varphi\left(\mathbf{x}^{*}\right)\right]\left[\nabla^{T} \varphi\left(\mathbf{x}^{*}\right)\right]\right)^{-1}\left[\nabla \varphi\left(\mathbf{x}^{*}\right)\right]\right\} \\
\cdot \nabla \mathcal{F}^{*}\left(\mathbf{x}^{*}\right)=\mathbf{0} \\
\left(\left[\nabla \varphi\left(\mathbf{x}^{*}\right)\right]\left[\nabla^{T} \varphi\left(\mathbf{x}^{*}\right)\right]\right)^{-1}\left[\nabla \varphi\left(\mathbf{x}^{*}\right)\right] \\
\nabla \mathscr{F}\left(\mathbf{x}^{*}\right) \geq 0
\end{gathered}
$$

Here $\left[\nabla \varphi\left(\mathbf{x}^{*}\right)\right]=\left[\frac{\partial \varphi\left(\mathbf{x}^{*}\right)}{\partial \mathbf{x}}\right]$ are the gradients of the problem (21) active constraints (satisfied as equalities, ie $\left.\varphi_{i}(\mathbf{x})=0, i \leq 1,2, \ldots, \zeta, i \in I\right)$. The investigations $[14,15]$ illustrate that the Kuhn-Tucker conditions (22)(23) are the strain compatibility equations. An illustration for the Kuhn-Tucker conditions is presented in Fig 2. The vector of gradient objective function $-\nabla \sigma\left(x^{*}\right)$ can be located inside in respect of cones being spanned by the gradient $\nabla \varphi_{1}\left(x^{*}\right), \nabla \varphi_{2}\left(x^{*}\right)$ of the active constraints $\varphi_{1}\left(\mathbf{x}^{*}\right)=0, \varphi_{2}\left(\mathbf{x}^{*}\right)=0$. The plastic multipliers correspond to the relationship (23) in the above-mentioned equations group, namely: 


$$
\lambda=\left(\left[\nabla \varphi\left(\mathbf{x}^{*}\right)\right]\left[\nabla^{T} \varphi\left(\mathbf{x}^{*}\right)\right]\right)^{-1}\left[\nabla \varphi\left(\mathbf{x}^{*}\right)\right] \nabla \mathscr{F}\left(\mathbf{x}^{*}\right)
$$$$
\lambda \geq 0 \text {. }
$$

The plastic multipliers are related to the yield conditions $\varphi_{i}(\mathbf{x}) \geq 0$ (these are the conditions (20) of the mathematical model (19)-(20)).

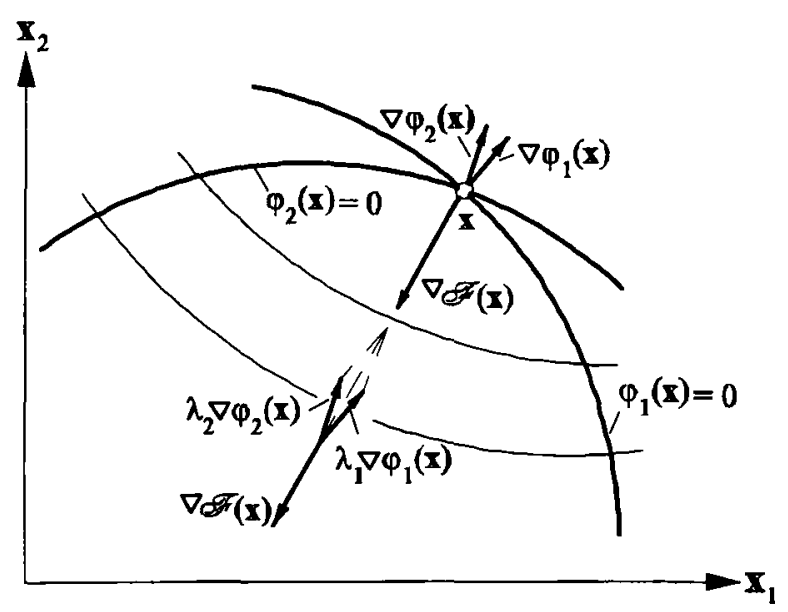

Fig 2. Illiustration for Kuhn-Tucker conditions

The plastic multipliers of the non-active yield conditions are equal to zero, therefore the mathematical programming complementarity conditions $\lambda^{T} \varphi\left(\mathbf{x}^{*}\right)=0$ are satisfied. The relationship (23) corresponds to the strain compatibility equations [14]:

$$
\begin{array}{r}
\left.\qquad B_{\theta}\right] \boldsymbol{\Theta}_{p}=\left[B_{r}\right] \mathbf{M}_{r}, \\
\text { where }\left[B_{\theta}\right]=\left[\left[A^{\prime \prime}\right]^{T}\left(\left[A^{\prime}\right]^{T}\right)^{-1},-[\mathrm{I}]\right], \\
{\left[B_{r}\right]=-\left[A^{\prime \prime}\right]^{T}\left(\left[A^{\prime}\right]^{T}\right)^{-1}\left[D^{\prime}\right]+\left[D^{\prime \prime}\right] .}
\end{array}
$$

Therefore, when applying the vectors $\mathrm{x}^{*}$ and $\lambda$, the dual problem variables can be obtained at once: and

$$
\mathbf{y}_{1}=\nabla \mathscr{F}\left(\mathbf{x}^{*}\right)=[\tilde{D}] \mathbf{x}^{*}, \quad \mathbf{y}_{2}=\left[\nabla \varphi\left(\mathbf{x}^{*}\right)\right]^{T} \lambda
$$

$$
\begin{gathered}
\mathbf{y}_{1}-\mathbf{y}_{2}=\mathbf{0}, \\
\lambda^{T} \varphi\left(\mathbf{x}^{*}\right)=\mathbf{0}, \\
\lambda \geq 0 .
\end{gathered}
$$

The section is ended by presenting the plasticity theory full equations system related to plates.

The full equations system contains all constraints (20) of the primary problem (19)-(20) in concert with the Kuhn-Tucker conditions (22)-(23) of the problems (19)-(20) optimal solution:

$$
\begin{gathered}
\varphi_{k l, j}=C_{k}-\left(\mathbf{M}_{e k l, j}+\left[B_{k l}\right]^{T} \mathbf{M}_{r}^{\prime \prime}\right)^{T}[\Phi]\left(\mathbf{M}_{e k l, j}+\right. \\
\left.+\left[B_{k l}\right]^{T} \mathbf{M}_{r}^{\prime \prime}\right) \geq 0,
\end{gathered}
$$

$$
\begin{gathered}
\boldsymbol{\varphi}_{j}=\left(\varphi_{k l, j}\right)^{T}=\varphi_{j}\left(\mathbf{M}_{j}\right), \quad \mathbf{M}_{j}=\mathbf{M}_{e, j}+\mathbf{M}_{r}, \\
\mathbf{M}_{r}=[B]^{T} \mathbf{M}_{r}^{\prime \prime} ; k \in K, \quad l \in L, \quad j \in J . \\
\sum_{j}\left[B_{\lambda_{j}} \mid \lambda_{j}=\left[B_{r}\right] \mathbf{M}_{r}, \quad\left\lfloor B_{\lambda_{j}}\right]=\left[B_{0}\right]\left[\nabla \boldsymbol{\varphi}_{j}\right],\right. \\
\lambda_{j} \geq \mathbf{0}, \quad \lambda_{j}=\left(\lambda_{k l, j}\right)^{T}, \\
\lambda_{j}^{T} \boldsymbol{\varphi}_{j}\left(\mathbf{M}_{j}\right)=\mathbf{0} .
\end{gathered}
$$

The vectors $\mathbf{M}_{r}^{\prime \prime}$ and $\lambda$ are unknowns of the problem (28). The plastic strains vector $\boldsymbol{\Theta}_{p}$ is not an independent variable as it is obtained by the formula (14):

$$
\begin{gathered}
\boldsymbol{\Theta}_{p k l}=2 \sum_{j} \lambda_{k l, j}[\Phi] \mathbf{M}_{k l, j}, \\
\mathbf{M}_{k l, j}=\mathbf{M}_{e k l, j}+\mathbf{M}_{r k l}, \quad \mathbf{M}_{r}=[B]^{T} \mathbf{M}_{r}, \\
{\left[B_{\theta}\right] \boldsymbol{\Theta}_{p}=\left[B_{r}\right] \mathbf{M}_{r}, \quad \boldsymbol{\Theta}_{p}=\left(\boldsymbol{\Theta}_{p k l}\right)^{T},} \\
k \in K, \quad l \in L, \quad j \in J .
\end{gathered}
$$

\section{Adapted plate load optimization mathematical models}

The adapted elastic-plastic plate is under consideration. The vector of limit bending moments $\mathbf{M}_{0}$ is prescribed. An elastic-plastic plate shakedown state is investigated. The quasi-static load is described by time $t$ independent load variation bounds $\mathbf{F}_{\text {sup }}, \mathbf{F}_{\text {inf }}$ (the load application points are fixed). The certain loading history is unknown but it is sloped by the load variation bounds $\mathbf{F}_{\text {inf }} \leq \mathbf{F}(t) \leq \mathbf{F}_{\text {sup }}$. Then the adapted elastic-plastic plate load optimization problem reads:

find the load variation bounds $\mathbf{F}_{\text {sup }}, \mathbf{F}_{\text {inf }}$, satisfying the prescribed optimality criterion max $\left\{\mathbf{T}_{\text {sup }}^{T} \mathbf{F}_{\text {sup }}+\mathbf{T}_{\text {inf }}^{T} \mathbf{F}_{\text {inf }}\right\}$ in concert with plate strength and stiffness requirements.

Here $\mathbf{T}_{\text {sup }}, \mathbf{T}_{\text {inf }}$ are the optimality criterion weight coefficient vectors.

The adapted plate is safe in respect of plastic collapse but possibly cannot satisfy certain maintenance conditions (as, for instance, the stiffness requirements). Therefore the strength requirements in concert with stiffness constraints are to be included into constraints of the adapted structure load variation bounds optimization mathematical model. The plate stiffness requirements include the residual strains $\Theta_{r}(t)$ and displacements $\mathbf{u}_{r}(t)$ dependable upon certain loading history. When the certain loading history is unknown, one can only define the shakedown state residual displacements variation bounds $\mathbf{u}_{r, \text { inf }} \leq \mathbf{u}_{r}(t) \leq \mathbf{u}_{r, \text { sup }}$ of the plate. Thus the adapted plate load optimization couples two main problems. The first problem aims to find the plate shakedown state ensuring residual bending moments $\mathbf{M}_{r}$. The problem is realized 
by solving the plate analysis problem (15)-(17). The second problem aims to verify the plate stiffness constraints $\mathbf{u}_{r, \min } \leq \mathbf{u}_{r, \text { inf }}, \quad \mathbf{u}_{r, \text { sup }} \leq \mathbf{u}_{r, \max }$. Here $\mathbf{u}_{r, \text { min }}$, $\mathbf{u}_{r, \max }$ are the prescribed codified plate deflections (fixed to ensure certain requirements for residual deformations). Therefore the residual deflections variation bounds $\mathbf{u}_{r, \text { inf }}, \mathbf{u}_{r, \text { sup }}$ evaluation problem needs to be solved [14]. It is obvious that plate load optimization problem at shakedown is not a classical mathematical programming problem. Note that the objective function $\max \left\{\mathbf{T}_{\text {sup }}^{T} \mathbf{F}_{\text {sup }}+\mathbf{T}_{\text {inf }}^{T} \mathbf{F}_{\text {inf }}\right\}$ magnitude identification problem combines both the above-mentioned related problems, namely the plate analysis problem (15)-(17) and that of the variation bounds $\mathbf{u}_{r \text {, inf }}, \mathbf{u}_{r \text {, sup }}$ identification. Therefore the problem solution is rather complicated.

The mathematical model formulation for the certain adapted construction load optimization problem is directly influenced by the method to evaluate the residual bending moments $\mathbf{M}_{r}$. This paper investigates two optimization problem formulations. The analysis problem (15) (17) in the first formulation is presented as the convex non-linear mathematical programming problem (21) or (19)-(20). Then the load optimization objective function $\max \left\{\mathbf{T}_{\text {sup }}^{T} \mathbf{F}_{\text {sup }}+\mathbf{T}_{\text {inf }}^{T} \mathbf{F}_{\text {inf }}\right\}$ is linear. The equations and relationships are stated according to the plasticity theory full equations system (28) in the second formulation of the load optimization problem. In this case the optimization problem objective function is non-linear, namely:

$$
\max \left\{\mathbf{T}_{\text {sup }}^{T} \mathbf{F}_{\text {sup }}+\mathbf{T}_{\text {inf }}^{T} \mathbf{F}_{\text {inf }}-\sum_{j} \boldsymbol{\lambda}_{j}^{T} \boldsymbol{\varphi}_{j}\left(\mathbf{M}_{j}\right)\right\} .
$$

According to the cyclic load definition $\mathbf{F}_{\text {inf }} \leq \mathbf{F}(t) \leq \mathbf{F}_{\text {sup }}$. In most cases the vectors $\mathbf{F}_{\text {inf }}$ and $\mathbf{F}_{\text {sup }}$ components are positive and negative respectively. For convenience it is assumed that $\mathbf{F}_{\text {inf }} \geq \mathbf{0}$ and $\mathbf{F}_{\text {sup }} \geq \mathbf{0}$ in our investigation. The latter assumption is applied when calculating the plate energy magnitudes and that of the plate stresses and strains both for elastic and plastic stages.

\subsection{First formulation of the problem mathematical model}

The first formulation is based on the problem (19)(20) solution. The actual adapted state residual bending moments $\mathbf{M}_{r}^{*}$ and optimal load variation bounds $\mathbf{F}_{\text {sup }}^{*}$, $\mathbf{F}_{i n f}^{*}$ are identified by means of the following problem solution:

find

$$
\max \left\{\mathbf{T}_{\text {sup }}^{T} \mathbf{F}_{\text {sup }}+\mathbf{T}_{\text {inf }}^{T} \mathbf{F}_{\text {inf }}\right\}=W
$$

subject to

$$
\min \frac{1}{2} \sum_{k} \mathbf{M}_{r}^{\prime \prime}[\widetilde{D}] \mathbf{M}_{r}^{\prime \prime}
$$

when

$$
\begin{gathered}
\boldsymbol{\varphi}_{k l, j}=C_{k}-\mathbf{M}_{k l, j}^{T}[\Phi] \mathbf{M}_{k l, j} \geq 0, \quad C_{k}=\left(M_{0 k}\right)^{2}, \\
\mathbf{M}_{k l, j}=\mathbf{M}_{e k l, j}+\mathbf{M}_{r k l}, \quad \mathbf{M}_{r}=[B]^{T} \mathbf{M}_{r}^{\prime \prime}, \\
k \in K, \quad l \in L, \quad j \in J . \\
\mathbf{F}_{i n f} \geq \mathbf{0}, \quad \mathbf{F}_{\text {sup }} \geq \mathbf{0},
\end{gathered}
$$

and

$$
\mathbf{u}_{r, \min } \leq \mathbf{u}_{r}=\left[H_{\theta}\right] \Theta_{p} \leq \mathbf{u}_{r, \max } .
$$

The vectors $\mathbf{F}_{\text {sup }}, \mathbf{F}_{\text {inf }}, \mathbf{M}_{r}^{\prime \prime}$ are the unknowns of the problem (29)-(30). The plastic strains $\Theta_{p}$ are identified by solving the problem (30). The satisfaction of the inequalities (31) $\mathbf{u}_{r, \min } \leq\left[H_{\theta}\right] \boldsymbol{\Theta}_{p} \leq \mathbf{u}_{r, \max }$ means that the adapted plate stiffness conditions are valid. As it was mentioned above, the vectors $\mathbf{u}_{r, \text { min }}, \mathbf{u}_{r, \max }$ are known prescribed residual displacements variation bounds. The inequality (31) contains the residual displacement influence matrix $\left[H_{\theta}\right]=\left([A][D]^{-1}[A]^{T}\right)^{-1}[A][D]^{-1}$. The plastic strains $\Theta_{p}=\left(\Theta_{p k l}\right)^{T}$ including the formula (31) are calculated applying the formula:

$$
\begin{gathered}
\boldsymbol{\Theta}_{p k l}=2 \sum_{j} \lambda_{k l, j}[\Phi] \mathbf{M}_{k l, j}, \\
\lambda_{k l, j}\left(C_{k}-\mathbf{M}_{k l, j}^{T}[\Phi] \mathbf{M}_{k l, j}\right)=0, \\
\lambda_{k l, j} \geq 0, \quad k \in K, \quad l \in L, \quad j \in J .
\end{gathered}
$$

As a result, the problem (29)-(31) contains the direct unknowns $\mathbf{F}_{\text {sup }}, \mathbf{F}_{\text {inf }}, \mathbf{M}_{r}^{\prime \prime}$ and that of the indirect unknown - the plastic multipliers vector $\lambda$. The last vector can be calculated by the formula (24). Having identified the plastic multipliers $\lambda_{j}(j \in J)$, the residual displacements $\mathbf{u}_{r}$ from the formula (31) are calculated applying the relation $\mathbf{u}_{r}=[H] \lambda$.

Note that during the plastic deformation process an unloading phenomenon of the cross-sections is possible. The unloading phenomenon means that at some deformation stages a yield condition is satisfied as equality, ie $\varphi_{k l, j}=0$ for $j$-th cross-section, in later deformation stages it changes to inequality $\varphi_{k l, j}>0$ and vice versa. For the real deformation process $\varphi_{k l, j}=0$, $\lambda_{k l, j} \varphi_{k l, j}=0, \lambda_{k l, j}>0$ and the plastic multiplier magnitude $\lambda_{k l, j}>0$ remains unchanged up to the loading process end. Unfortunately, the analysis problem mathematical model (19)-(20) does not enable to represent directly the deformation process per time $t$. It is obvious that the mathematical programming complementarity condition (26) $\lambda^{T} \varphi\left(\mathbf{M}^{*}\right)=0$ does not allow to evaluate the unloading phenomenon. Taking into account the possibility of unloading, the inequality (31) should be transformed to:

$$
u_{r i, \min } \leq \min \left[H_{i}\right] \lambda=u_{r i, i n f},
$$




$$
\begin{gathered}
\max \left[H_{i}\right] \lambda=u_{r i, \text { sup }} \leq u_{r i, \max }, \\
i=1,2, \ldots, m
\end{gathered}
$$

Here the index $i$ is related to the deflection vector $\mathbf{u}$ components $u_{1}, u_{2}, \ldots, u_{m}$. It is clear that the number of constrained displacements should be less than the number $m$.

An evaluation of residual displacement bounds $\mathbf{u}_{r, \text { inf }}, \mathbf{u}_{r, \text { sup }}$ is a rather complicated problem [14, 15]. In this paper the bounds $u_{r i, s u p}, u_{r i \text { inf }}$ are identified by solving the problem:

find

$$
\max _{\min }\left[H_{i}\right] \tilde{\lambda}=\left[\begin{array}{l}
u_{r i, s u p} \\
u_{r i, i n f}
\end{array}\right]
$$

subject to

$$
\begin{gathered}
{\left[B_{\lambda}\right] \tilde{\boldsymbol{\lambda}}=\left[B_{r}\right] \mathbf{M}_{r}^{*}, \quad \tilde{\lambda} \geq 0,} \\
\tilde{\lambda}=\left(\tilde{\boldsymbol{\lambda}}_{j}\right), \quad \sum_{j} \tilde{\boldsymbol{\Lambda}}_{j}^{T} \tilde{\mathbf{C}} \leq \tilde{D}_{\max } .
\end{gathered}
$$

During the structural shakedown process the energy is dissipated. The upper bound of the dissipated energy magnitude $D_{\max }$ can be calculated by the Koiter's suggested formula [7]. The fictitious structure method proposed in the investigation $[14,15]$ allows to evaluate more exactly the energy dissipation bound magnitude $\widetilde{D}_{\max }\left(\widetilde{\lambda}\right.$ notation is compatible with notation $\left.\widetilde{D}_{\max }\right)$. An extended investigation of the problem (34)-(35) is presented in [14].

The problem (19)-(20) can also be used for the stress and strain field evaluation during the real plate deformation process. Then the problem (30) is solved via iterative procedures for each objective function (29) increment $\Delta W$ (for further details concerning the algorithm the reader is referred to Section 6). But note for the latter case the displacement bounds $\mathbf{u}_{r, \text { inf }}, \mathbf{u}_{r \text {, sup }}$ evaluation problem (34)-(35) solution is necessary.

\subsection{Second formulation of the problem mathematical model}

The optimization problem second formulation is stated by applying the plasticity theory full equations system (28) and reads as follows:

find

$$
\max \left\{\mathbf{T}_{\text {sup }}^{T} \mathbf{F}_{\text {sup }}+\mathbf{T}_{\text {inf }}^{T} \mathbf{F}_{\text {inf }}\right\}=W
$$

subject to

$$
\begin{gathered}
\varphi_{k l, j}=C_{k}-\mathbf{M}_{k l, j}^{T}[\Phi] \mathbf{M}_{k l, j} \geq 0, \quad C_{k}=\left(M_{0 k}\right)^{2} \\
\mathbf{M}_{k l, j}=\mathbf{M}_{e k l, j}+\mathbf{M}_{r k l}
\end{gathered}
$$

$$
\begin{gathered}
{\left[B_{\theta}\right] \boldsymbol{\Theta}_{p}=\left[B_{r}\right] \mathbf{M}_{r}, \quad \boldsymbol{\Theta}_{p}=\left(\boldsymbol{\Theta}_{p k l}\right)^{T},} \\
\boldsymbol{\Theta}_{p k l}=2 \sum_{j} \lambda_{k l, j}[\Phi] \mathbf{M}_{k l, j}, \\
\lambda_{k l, j} \geq 0, \quad \lambda_{k l, j} \varphi_{k l, j}=0, \quad k \in K, \quad l \in L, \quad j \in J ; \\
\mathbf{F}_{i n f} \geq \mathbf{0}, \quad \mathbf{F}_{s u p} \geq \mathbf{0}
\end{gathered}
$$

and

$$
\mathbf{u}_{r, \min } \leq\left[H_{\theta}\right] \boldsymbol{\Theta}_{p} \leq \mathbf{u}_{r, \max } .
$$

The vectors $\mathbf{F}_{\text {sup }}, \mathbf{F}_{\text {inf }}, \mathbf{M}_{r}$ (taking into consideration $\mathbf{M}_{r}=[B]^{T} \mathbf{M}_{r}^{\prime \prime}$ ) and $\lambda_{j}$ are the problem of (36)-(38) unknowns.

In investigation [26] the complementarity conditions were proposed to include into objective function of the optimization problem. Then the rewritten adapted plate load optimization problem (36)-(38) reads:

find

$$
\begin{gathered}
\max \left\{\mathbf{T}_{\text {sup }}^{T} \mathbf{F}_{\text {sup }}+\mathbf{T}_{\text {inf }}^{T} \mathbf{F}_{\text {inf }}-\sum_{k l, j} \lambda_{k l, j}\left[C_{k}-\right.\right. \\
\left.\left.-\mathbf{M}_{k l, j}^{T}[\Phi] \mathbf{M}_{k l, j}\right]\right\}=W
\end{gathered}
$$

subject to

$$
\begin{gathered}
\varphi_{k l, j}=C_{k}-\mathbf{M}_{k l, j}^{T}[\Phi] \mathbf{M}_{k l, j} \geq 0, \quad C_{k}=\left(M_{0 k}\right)^{2}, \\
\mathbf{M}_{k l, j}=\mathbf{M}_{e k l, j}+\mathbf{M}_{r k l}, \\
{\left[B_{\theta}\right] \boldsymbol{\Theta}_{p}=\left[B_{r}\right] \mathbf{M}_{r}, \quad \boldsymbol{\Theta}_{p}=\left(\boldsymbol{\Theta}_{p k l}\right)^{T},} \\
\boldsymbol{\Theta}_{p k l}=2 \sum_{j} \lambda_{k l, j}[\Phi] \mathbf{M}_{k l, j}, \\
\lambda_{k l, j} \geq 0, \quad k \in K, \quad l \in L, \quad j \in J ; \\
\mathbf{F}_{i n f} \geq \mathbf{0}, \quad \mathbf{F}_{s u p} \geq \mathbf{0}
\end{gathered}
$$

and

$$
\mathbf{u}_{r, \min } \leq\left[H_{\theta}\right] \Theta_{p} \leq \mathbf{u}_{r, \max }
$$

The vectors $\mathbf{F}_{\text {sup }}, \mathbf{F}_{\text {inf }}, \mathbf{M}_{r}^{\prime \prime}, \lambda_{j}$ are the unknowns of the problem (36)-(38). By analogy with the problem (29)-(31) case it is necessary to take into account the possible unloading when solving the problem (34)-(35).

\section{Algorithm for solution of the load optimization problem}

An algorithm for solving of the optimization problem first formulation (29)-(31) is to be presented in detail. The algorithm statement is based on the Rozen project gradient method and its mechanical methods 
optimality criterions interpretation, already described in Section 4. The problem (29)-(31) solution is replaced by means of the three different problems solution in a consecutive order. The solution process is iterative.

The first problem is the optimal load variation bounds $\mathbf{F}_{\text {sup }}^{*}, \mathbf{F}_{\text {inf }}^{*}$ determination for plastic collapse. The mathematical model of this problem reads:

find

$$
\max \left\{\mathbf{T}_{\text {sup }}^{T} \mathbf{F}_{\text {sup }}+\mathbf{T}_{\text {inf }}^{T} \mathbf{F}_{\text {inf }}\right\}=W
$$

subject to

$$
\begin{gathered}
\sum_{k}\left[A_{k}\right] \mathbf{M}_{r k}=\mathbf{0}, \\
\varphi_{k l, j}=C_{k}-\mathbf{M}_{k l, j}^{T}[\Phi] \mathbf{M}_{k l, j} \geq 0, \quad C_{k}=\left(M_{0 k}\right)^{2}, \\
k \in K, \quad l \in L, \quad j \in J \\
\mathbf{F}_{\text {inf }} \geq \mathbf{0}, \quad \mathbf{F}_{\text {sup }} \geq \mathbf{0} .
\end{gathered}
$$

The load variation bounds $\mathbf{F}_{\text {sup }}, \mathbf{F}_{\text {inf }}$ and residual bending moments $\mathbf{M}_{r}$ (or after application of the relationship (18), the $\mathbf{M}_{r}^{\prime \prime}$ ) are the unknowns of the problem (43)-(45). Although the objective functions of the problems (29)-(31) and (43)-(45) are similar, one must find that the physical meaning of the optimality criterion coefficients $\mathbf{T}_{\text {sup }}, \mathbf{T}_{\text {inf }}$ is different. The coefficients in the objective function (43) mean the extreme magnitudes of residual displacements rates. Thus the objective function (43) means the work of external forces per time unit, ie the power of external loads $W$. The bounds $\mathbf{F}_{\text {sup }}^{*}, \mathbf{F}_{\text {inf }}^{*}$, being identified by solution of the problem (43)-(45), correspond to the cyclic plastic collapse case. At the moment, just before the cyclic plastic collapse moment, the plate shakedown state of residual deflections magnitudes do not satisfy the stiffness constraints (31). It is obvious that the more strict constraints (33), resulting from the problem (34)-(35) solution, would fail. The limiting magnitudes of residual displacements $\mathbf{u}_{r, \min }, \mathbf{u}_{r, \max }$ ensuring the maintenance conditions cannot exceed the magnitudes to be reached just before the plastic failure. The each problem (43)-(45) $v$-th solution stage duration is limited by the external forces power magnitude $\Delta W^{\nu}$. The load variation bounds at the end of the $v$-th stage are denoted via $\mathbf{F}_{\text {inf }}^{v}$. The load vectors $\mathbf{F}_{\text {sup }}^{v}, \mathbf{F}_{\text {inf }}^{v}$ are applied when solving the problem to identify the residual bending moments $\mathbf{M}_{r}^{v}$. It is necessary because of the existence of the other distribution of residual bending moments resulting from the less magnitude of the objective function (15) in respect of the magnitude $\frac{1}{2} \sum_{k} \mathbf{M}_{r k}^{v T}\left[D_{k}\right] \mathbf{M}_{r k}^{v}$. This phenomenon will be described in detail.

The second problem is an analysis of shakedown state residual bending moments $\mathbf{M}_{r}^{\nu}$ by means of the problem (15)-(17) or the problem (19)-(20) solution.
Note that the problem is to be solved for the each $v$-th stage load magnitudes $\mathbf{F}_{\text {sup }}^{v}, \mathbf{F}_{\text {inf }}^{v}$. An admissible solution of the analysis problem (19)-(20) is received by means of the simple application of the problem (43)(45) $v$-th stage optimal solution. The problem (43)-(45) $v$-th stage solution $\mathbf{F}_{\text {sup }}^{v}, \mathbf{F}_{\text {inf }}^{v}$ and $\mathbf{M}_{r}^{v}$ belongs to the admissible set of variables $\mathbf{x}$, ie: $\mathscr{Z}=\left\{\mathbf{x} \mid \boldsymbol{\varphi}_{i}(\mathbf{x}) \geq 0\right.$ for $\left.i=1,2, \ldots, \zeta\right\}$. The vector $\mathbf{M}_{r}^{* v}$ is the analysis problem optimal solution. The plastic multipliers $\lambda^{* \nu}$ are calculated applying the Rozen optimality criterion formula (24). The plastic strains $\Theta_{p}^{* v}$ and plastic multipliers are related via formula (14):

$$
\Theta_{p k l}^{* \nu}=2 \sum_{j} \lambda_{k l, j}^{* \nu}[\Phi] \mathbf{M}_{k l, j}^{* \nu}, \quad k \in K, \quad l \in L, \quad j \in J .
$$

The third problem is that of the verification of the plate stiffness conditions $\mathbf{u}_{r, \min } \leq\left[H_{\theta}\right] \Theta_{p}^{* \nu} \leq \mathbf{u}_{r, \max }$. When these conditions are satisfied, the following $v$-th step of the problem (43)(45) is executed. When the conditions (31) are not satisfied, the increment of objective function (43) $\Delta W^{v}$ is reduced and the problem (43)-(45) is resolved. Following the above-mentioned procedures the satisfaction of all constraints $\mathbf{u}_{r, \min } \leq[H] \Theta_{p}^{* \boldsymbol{v}} \leq \mathbf{u}_{r, \max }$ is achieved and the vectors $\mathbf{F}_{\text {sup }}^{* v}, \mathbf{F}_{\text {inf }}^{* \mathrm{v}}$ are said to be the rational solution of the problem (43)-(45).

However, verification of the stiffness conditions $\mathbf{u}_{r, \min } \leq[H] \boldsymbol{\Theta}_{p} \leq \mathbf{u}_{r, \max }$ in the cyclic load case can be insufficient. When identifying the plate stress state, one must evaluate the possible unloading phenomenon during the shakedown process. Therefore instead of the verification of the conditions (31) the more strict verification (33) is introduced. To ensure such a verification, the problem (34)-(35) is to be solved. Only then an optimal adapted plate loads rational variation bounds $\mathbf{F}_{\text {sup }}^{*}$, $\mathbf{F}_{\text {inf }}^{*}$ are to be obtained. The problem (29)-(31) solution algorithm scheme is presented in Fig 3. An algorithm application is illustrated via numerical examples of Section 6.

The feature of the presented algorithm is that the stiffness conditions are verified only at the $v$-th stage end of the bounds $\mathbf{F}_{\text {sup }}^{*}, \mathbf{F}_{\text {inf }}^{*}$ search. This feature is predetermined by the load optimization mathematical model formulation as the load optimization problem mathematical model second formulation (39)-(42). Variables are not only the $\mathbf{F}_{\text {sup }}, \mathbf{F}_{\text {inf }}, \mathbf{M}_{r}^{\prime \prime}$ but also the $\lambda$. This feature can be ignored when determining the rational solution $\mathbf{F}_{\text {sup }}^{* v}, \mathbf{F}_{\text {inf }}^{* v}$ by means of the above-mentioned formulation. But find that the complicating solution factor in this case is the non-linearity of the compatibility equations. Note that by analogy with the problem (29)-(31) case, the solution of the problem (39)-(41) requires to verify the stiffness conditions via solution of the (34)(35) problem. The problem (39)-(42) solution algorithm scheme is presented in Fig 4. 


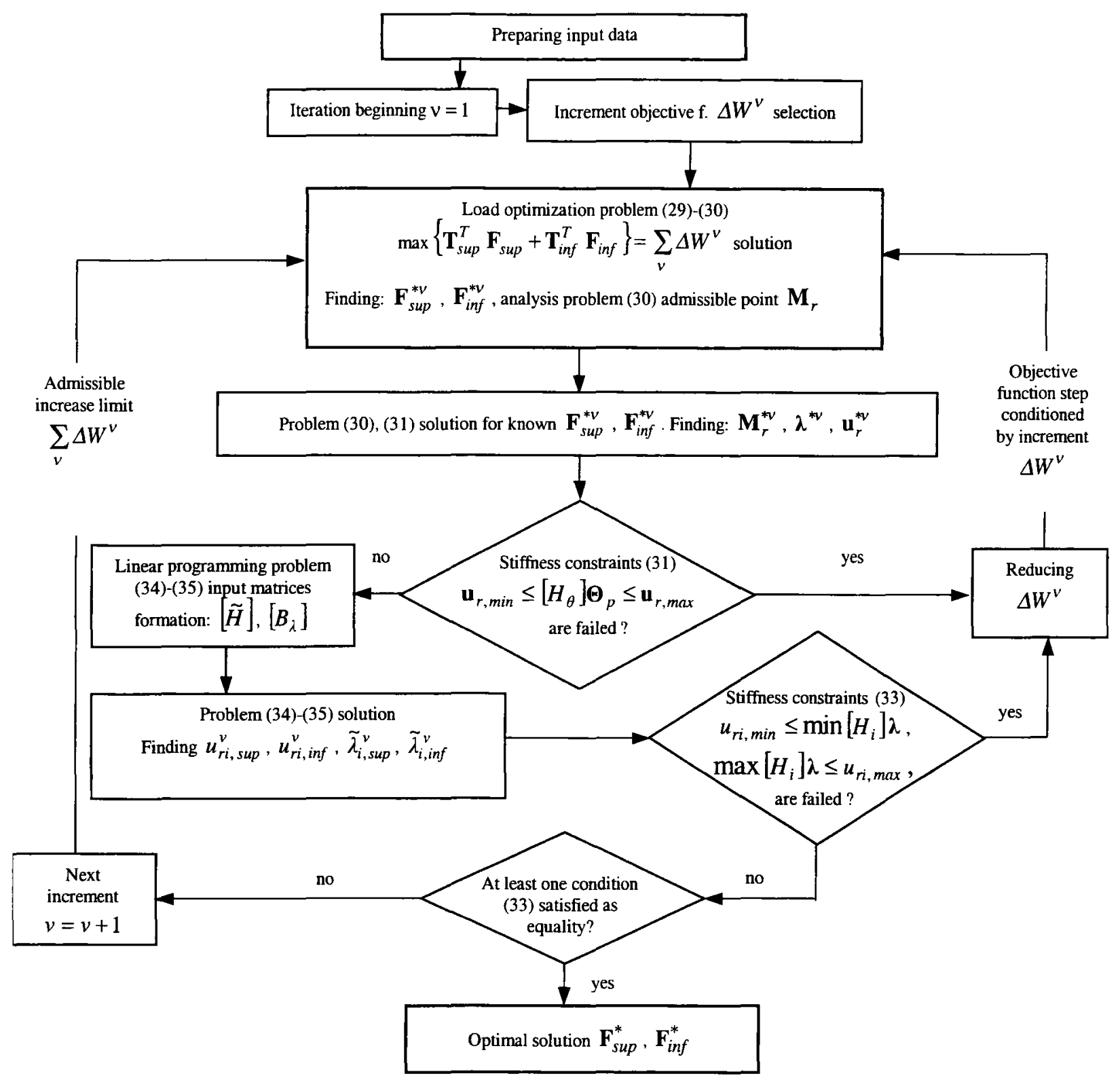

Fig 3. Solution algorithm of the problem (29)-(31)

\section{Numerical examples of the plate load optimization problem}

The shakedown plate load optimization problem is realized via the mathematical model first formulation (29)-(31). The presented examples are simple and dedicated only to illustrate the proposed solution method. A perfectly elastic-plastic circular radii $R$ plate is under consideration (Fig 5). The hinge-fixed plate is subjected to the uniformly distributed of intensity $q$ load. The load intensity magnitude $q=1$ results in the following elastic bending moments:

$\mathbf{M}_{e}=\left[\begin{array}{llllll}0.20625 & 0.20625 & 0.19366 & 0.19883 & 0.15469\end{array}\right.$

$\begin{array}{llllll}0.17656 & 0.15469 & 0.17656 & 0.09023 & 0.13945 & 0.0\end{array}$
$0.08750]^{T}$. The plate limit bending moment $M_{0}=$ const is prescribed, the Poisson's ratio is equal to 0.3 . The equilibrium finite elements are applied for discretization.

First, the load $q$ maximum variation interval max $\left(q_{\text {sup }}+q_{\text {inf }}\right)$ is identified by solving the problem (43)(45). The alternating plasticity collapse results in magnitudes $q_{\text {sup }}=-q_{\text {inf }}=4.8485 M_{0} R^{-2}$. The result practically coincide with the J. König's received analytical solutions [7] when the plate is discretized by 10 equilibrium finite elements $(k=0)$. 


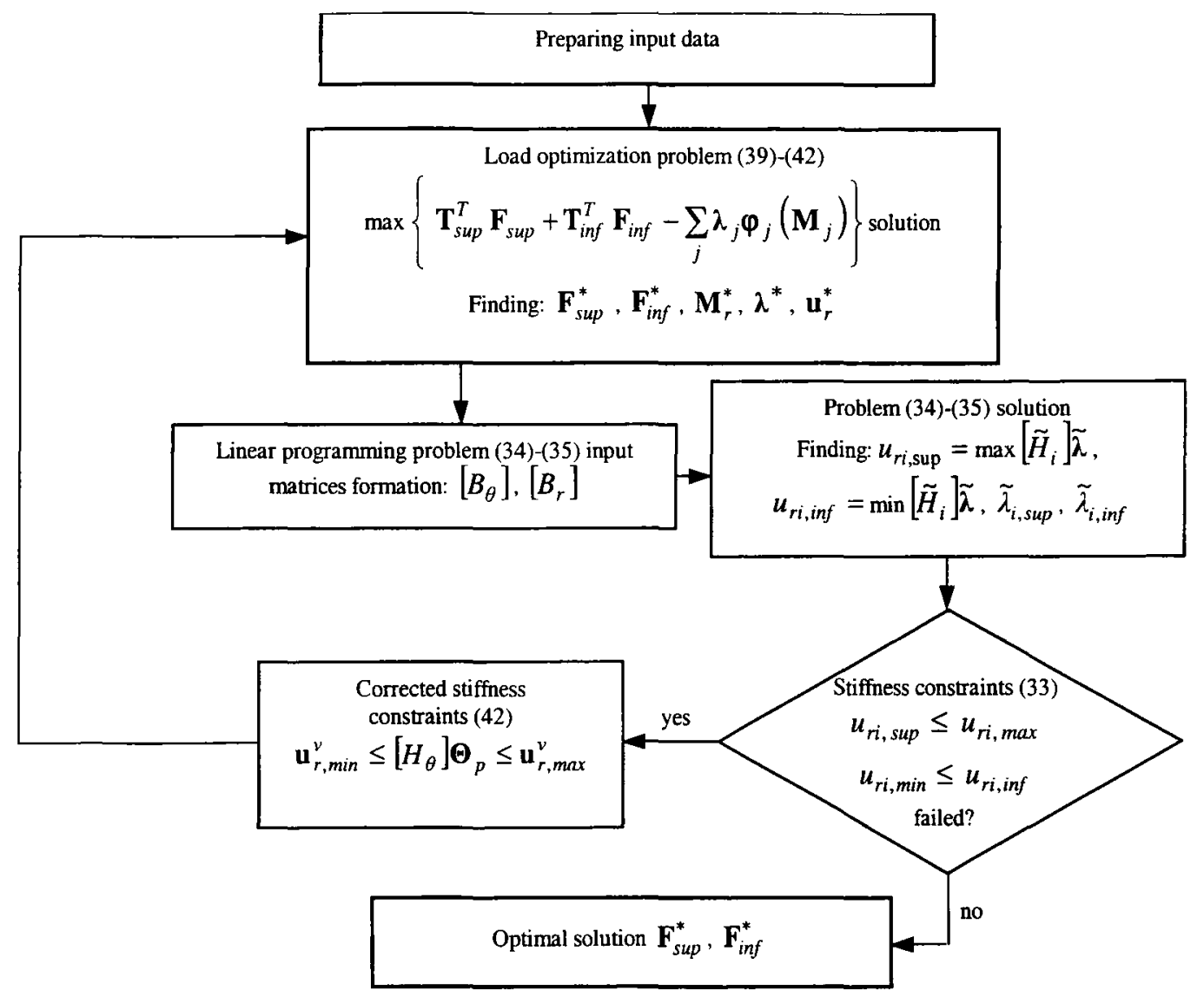

Fig 4. Solution algorithm of the problem (39)-(42)

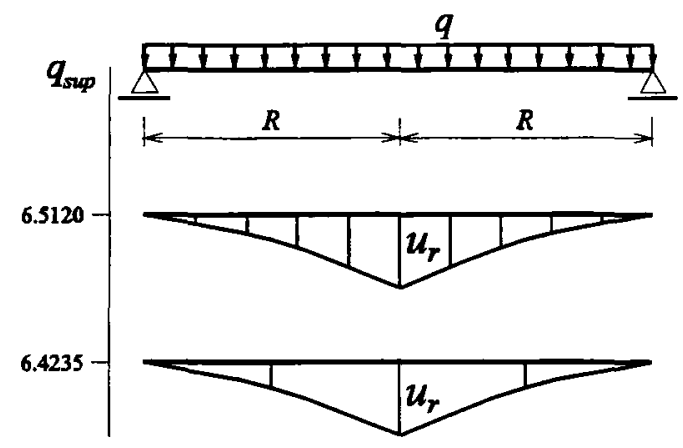

Fig 5. Plate design

An alternating plasticity case allows the existence of many different statically possible plate residual bending moments distributions. If $q_{\text {sup }}=-q_{\text {inf }}$, the residual bending moments $\mathbf{M}_{r}=\mathbf{0}$. The problem $\max q_{\text {sup }}$, for $0 \leq q \leq q_{\text {sup }}$ and $p=1$ solution results in $q_{\text {sup }}=6.5120 M_{0} R^{-2}$. The equilibrium finite elements are sufficiently accurate [24], therefore the only by two elements discretized plate solution results in the load limit $q_{\text {sup }}=6.4235 M_{0} R^{-2}$ when deflections were not limited up to the cyclic plastic failure. Such a type of problem is often used to test the discretization programme and methods. For more details of the mathematical programming methods application to calculate the structures af- fected by the cyclic loading, the reader is referred to the investigations $[1,2,5,7,9$, etc $]$ of the reference list.

Example 1. The plate shakedown state is under consideration. The load optimization problem $\max q_{\text {sup }}$ is realized via the mathematical model (29)-(31). An admissible plate deflection in the centre is $u_{r 1, \max }=1.80 M_{0} R / \mathscr{K}$, the stiffness condition (31) is formulated as $0 \leq u_{r 1} \leq u_{r 1, \max }$. The residual deflection bound $u_{r 1 \text {,sup }}$ evaluation method will be presented in detail, providing the solutions for the case $k=2$ (find that the larger number of finite elements significantly increases the linear programming problem (34)-(35) dimensions see Table 1). The plate discrete model parameters are: $s=6$ nodes with total degree of freedom $m=8$.

As it has been noted in Section 6, the problem (29)(31) solution is iterative and followed by solution of the three problems in the consecutive order for each stage. The problem (43)-(45) first stage $v=1$ solution results in the magnitude $q^{v=1}=5.5853 M_{0} R^{-2}$. Due to the problem (29)-(31) solution algorithm block-scheme (Fig 3) the second problem solution is to be followed further.

The second problem - the plate shakedown state analysis problem (30) (or (15)-(17)) now is solved for the previously identified load parameter load $q^{\nu=1}=5.5853 M_{0} R^{-2}$. A stage $v=1$ solution of the 
Table 2. $u_{r 1, \text { sup }}$ calculation problem $q^{v=1}=5.5853 M_{0} R^{-}$

\begin{tabular}{|c|c|c|c|c|c|c|c|c|}
\hline able 1. Structure & em (34)- & $\bar{\lambda}_{1}^{v}$ & $\bar{\lambda}_{2}^{v}$ & $\overline{\bar{\lambda}_{3}^{v}}$ & $\overline{\lambda_{4}^{v}}$ & $\bar{\lambda}_{5}^{v}$ & $\overline{\lambda_{6}^{v}}$ & \\
\hline \multirow{4}{*}[B_{\lambda}]{$\bar{\lambda}$} & \multirow{4}{*}{$=\left[B_{r}\right] \mathbf{M}_{r}^{*}$} & -1.9992 & -0.3652 & -0.0845 & 0.1094 & 0.2434 & & $=-0.7316$ \\
\hline & & & -1.7027 & -0.3380 & 0.4372 & 0.9734 & & $=-1.9633$ \\
\hline & & & 0.0730 & -1.5062 & 0.1092 & 0.2433 & & $=-0.0261$ \\
\hline & & & & & 0.6245 & 0.1664 & -1.0088 & $=0.0015$ \\
\hline \multirow{6}{*}{$-\bar{\lambda}$} & \multirow{6}{*}{$\leq 0$} & -1.0 & & & & & & $\leq 0.0$ \\
\hline & & & -1.0 & & & & & $\leq 0.0$ \\
\hline & & & & -1.0 & & & & $\leq 0.0$ \\
\hline & & & & & -1.0 & & & $\leq 0.0$ \\
\hline & & & & & & -1.0 & & $\leq 0.0$ \\
\hline & & & & & & & -1.0 & $\leq 0.0$ \\
\hline$\overline{\bar{\lambda}}^{T} \mathbf{C}$ & $\leq D_{\max }$ & 1.0 & 1.0 & 1.0 & 1.0 & 1.0 & 1.0 & $\leq 1.3661$ \\
\hline $\max \mathbf{u}_{r}=[H] \bar{\lambda}$ & Objective f. & 0.7325 & 0.3500 & 0.2410 & 0.2131 & 0.1025 & 0.0620 & Objective f. \\
\hline $\bar{\lambda}^{*}$ & $u_{r i, s u p}$ & 0.1549 & 1.1392 & 0.0727 & 0.0023 & & & 0.5303 \\
\hline
\end{tabular}

problem results in the actual distribution of residual bending moments $\mathbf{M}_{r}^{* v=1}$ :

$$
\left[\begin{array}{cc}
M_{r \rho} & M_{r \theta} \\
-0.1523 & -0.1523 \\
-0.1229 & -0.0728 \\
-0.0521 & 0.1307 \\
-0.0521 & 0.0443 \\
-0.1499 & 0.0632 \\
0.0 & 0.0157
\end{array}\right]
$$

and that of the vector of plastic multipliers $\lambda^{* \nu=1}=\left[\begin{array}{llllll}0.1549 & 1.1387 & 0.0725 & 0.0 & 0.0 & 0.0\end{array}\right]^{T}$. The zero magnitude plastic multipliers are related to the nonactive yield conditions. The yield conditions are satisfied as equalities in the 1, 2, 3 nodes. For the $v$-th stage $D_{\max }=\left(\lambda^{* \nu}\right)^{T} \mathrm{C}=1.3661 \quad M_{0}^{2} R / \delta \mathscr{K}$. The second and third problems are related via the plastic multipliers vector $\lambda^{* \nu}$.

The third problem is verification of the stiffness conditions (31) $0 \leq u_{r 1} \leq u_{r 1, \max }$. The residual displacement magnitude $u_{r 1}=0.5295 M_{0} R / \circlearrowright \mathscr{K}$ is obtained from the vector $\mathbf{u}_{r}^{* \nu=1}=[H] \lambda^{* \nu=1}=\left[\begin{array}{ll}0.5295 & 0.5683\end{array}\right.$ $\left.\begin{array}{llllll}0.2635 & 0.6473 & 0.2485 & 0.2555 & -0.0155 & 0.4366\end{array}\right]^{T}$. The obtained results yield the satisfaction of stiffness condition (31) $0 \leq 0.5295 \leq 1.80$.

If the unloading phenomenon appears, the stiffness condition (31) cannot be satisfied. Therefore the stiffness condition (31) is changed by a more strict one $0 \leq u_{r 1, \text { sup }} \leq 1.80$. The upper deflection bound $u_{r 1, \text { sup }}$ when $\widetilde{\mathbf{C}}=\mathbf{C}$ is calculated applying the mathematical model (34)-(35), which is graphically presented in Table 1. The plate deflection bound $u_{r 1, \text { sup }}$ evaluation problem is presented in Table 2 . The vector $\bar{\lambda}^{\nu}$ is the unknown of the problem. The model includes the compatibility equations $\left[B_{\lambda}\right] \bar{\lambda}^{v}=\left[B_{r}\right] \mathbf{M}_{r}^{* v=1}$, containing an optimal solution (46) of the analysis problem (15)-(17).
The objective function of the problem is $u_{r 1, \text { sup }}=\max \left[H_{1}\right] \bar{\lambda}^{v=1}$. An extended form of the objective function $\left[H_{1}\right] \bar{\lambda}^{\nu=1}=0.7325 \bar{\lambda}_{1}^{v}+0.3500 \bar{\lambda}_{2}^{v}+$ $+0.2410 \bar{\lambda}_{3}^{v}+0.2131 \bar{\lambda}_{4}^{v}+0.1025 \bar{\lambda}_{5}+0.0620 \bar{\lambda}_{6}$ is located in the one of the bottom lines of Table 2 . The bottom line of Table 2 contains the vector of plastic multipliers

$\bar{\lambda}^{* \nu=1}=\left[\begin{array}{llllll}0.1549 & 1.1392 & 0.0727 & 0.0023 & 0.0 & 0.0\end{array}\right]^{T}$ and that of the objective function optimal magnitude $u_{r 1, \text { sup }}=0.5303 M_{0} R / \mathcal{K}$ respectively. As the problem (34)-(35) is not degenerated $u_{r 1}=u_{r 1, \text { sup }}$ and the first stage is finished by identification of the $u_{r 1, \text { sup }}$ magnitude.

After a certain number solution iterations the problem (29)-(31) convergates to the optimal solution reading as follows: $q_{\text {sup }}^{*}=6.0512 M_{0} R^{-2}, \lambda^{*}=\left[\begin{array}{ll}0.1523 & 1.1392\end{array}\right.$ $\left.\begin{array}{llll}0.0073 & 0.0023 & 0.0 & 0.0\end{array}\right]^{T}, \quad \mathbf{u}_{r}^{*}=\left[\begin{array}{lll}1.7374 & 1.8419\end{array}\right.$ $\left.\begin{array}{llllll}1.0739 & 2.0350 & 1.0056 & 0.8107 & 0.0599 & 1.7079\end{array}\right]^{T}$.

Example 2. The previously investigated circular plate is subjected to the cyclic uniformly distributed load $q\left(0 \leq q \leq q_{\text {sup }}\right)$ and that of onto the outer contour applied uniformly distributed bending moment $M$ ( $0 \leq M \leq M_{\text {sup }}$ ) (Table 5, scheme a). The extreme load variation bounds $q_{\text {sup }}$ and $M_{\text {sup }}$ are to be determined taking into account the plate middle point deflection limitation: $u_{r 1} \leq u_{r 1, \max }=0.84 M_{0} R / \mathscr{K}$. The problem is solved applying the mathematical model (29)-(31). The solution process is iterative following the three problems in a consecutive order solutions. The first problem (43)(45) yield conditions are verified for all $(p=3)$ elastic stresses polyhedron apexes. The pseudoelastic bending moments $\mathbf{M}_{e 1}, \mathbf{M}_{e 2}, \mathbf{M}_{e 3}$ result from the load combinations $q ; M ; q$ and $M$, separate or simultaneous actions respectively.

Then the elastic plate solutions are denoted via $\mathbf{M}_{1}=\mathbf{M}_{e 1}+\mathbf{M}_{r}, \quad \mathbf{M}_{2}=\mathbf{M}_{e 2}+\mathbf{M}_{r}, \quad \mathbf{M}_{3}=\mathbf{M}_{e 3}+\mathbf{M}_{r}$. For instance, the first node yield conditions applying the (12) formula read: 


$$
\mathbf{M}_{11, j}^{T}[\Phi] \mathbf{M}_{11, j} \leq\left(M_{01}\right)^{2}
$$

or

$$
\left(\mathbf{M}_{e, 11, j}+\mathbf{M}_{r, 11}\right)^{T}[\Phi]\left(\mathbf{M}_{e, 11, j}+\mathbf{M}_{r, 11}\right) \leq\left(M_{01}\right)^{2},
$$

$$
j=1,2,3 \text {. }
$$

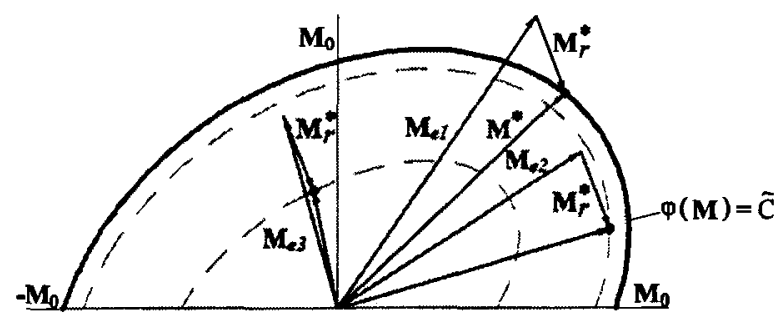

Fig 6. Yield conditions for the Example 2 problem

Applying the elastic bending moments $\mathbf{M}_{e 1}, \mathbf{M}_{e 2}$, $\mathbf{M}_{e 3}$, presented in Table 3, the first node yield conditions read:

$$
\begin{aligned}
& \left(0.20625 q+M_{r p, 11}\right)^{2}-\left(0.20625 q+M_{r p, 11}\right)(0.20625 q+ \\
& \left.+M_{r \theta, 11}\right)+\left(0.20625 \cdot q+M_{r \theta, 11}\right)^{2} \leq\left(M_{0}\right)^{2}, \\
& \left(-1.0 M+M_{r p, 11}\right)^{2}-\left(-1.0 M+M_{r p, 11}\right)\left(-1.0 M+M_{r \theta, 11}\right)+ \\
& +\left(-1.0 M+M_{r \theta, 11}\right)^{2} \leq\left(M_{0}\right)^{2}, \\
& \left(0.20625 q-1.0 M+M_{r p, 11}\right)^{2}-(0.20625 q-1.0 M+ \\
& \left.+M_{r p, 11}\right)\left(0.20625 q-1.0 M+M_{r \theta, 11}\right)+(0.20625 q-1.0 M+ \\
& \left.+M_{r \theta, 11}\right)^{2} \leq\left(M_{0}\right)^{2} .
\end{aligned}
$$

The end of first stage $(v=1)$ solution results in the values: $q^{v=1}=5.3881 M_{0} R^{-2}, M^{v=1}=0.8882 M_{0}$.

The vector of residual bending moments reads:

$$
\mathbf{M}_{r}^{* v=1}=\left[\begin{array}{cc}
M_{r p} & M_{r \theta} \\
-0.1112 & -0.1112 \\
-0.0744 & -0.0417 \\
-0.0461 & 0.0019 \\
-0.0461 & 0.0461 \\
-0.1151 & 0.0576 \\
0.0 & 0.0
\end{array}\right] \text {. }
$$

All 18 yield conditions of the discrete plate model will be verified. Only the first node yield condition is satisfied as equality. Consequently, the actual residual bending moments magnitudes $M_{r p, 11}=0.1112$, $M_{r \theta, 11}=0.1112$ are fixed only in this cross-section. The whole structure actual residual bending moments are identified by solving the analysis problem, stated applying a complementary deformation energy principle.

The second - the plate state analysis problem (15)(17) is realized applying the previously obtained values $q_{\text {sup }}^{v=1}=5.3881 M_{0} R^{-2}, M_{\text {sup }}^{v=1}=0.8882 M_{0}$. The solution of the analysis problem results in the actual distribution of the plate residual bending moments:

$$
\mathbf{M}_{r}^{* v=1}=\left[\begin{array}{cc}
M_{r \rho} & M_{r \theta} \\
-0.1112 & -0.1112 \\
-0.0789 & -0.0376 \\
-0.0283 & 0.0918 \\
-0.0283 & 0.0239 \\
-0.0081 & 0.0343 \\
0.0 & 0.0087
\end{array}\right] .
$$

The plate analysis problem (15)-(17) was realized applying the computer programme "ELROZEN", stated on the basis of the Rozen project gradient method application. The optimality criterions mathematical-mechanical interpretation (22)-(23) resulted in the following vector of plastic multipliers:

$$
\begin{aligned}
& \lambda^{*}=\left[\begin{array}{llllllllll}
0.1064 & 0.6300 & 0.0 & 0.0 & 0.0 & 0.0 & 0.0 & 0.0 & 0.0 \\
0.0 & 0.0 & 0.0 & 0.0 & 0.0 & 0.0 & 0.0 & 0.0 & 0.0
\end{array}\right]^{T} .
\end{aligned}
$$

Table 3. Vectors $\mathbf{M}_{e 1}, \mathbf{M}_{e 2}, \mathbf{M}_{e 3}$

\begin{tabular}{|c|c|c|c|c|c|}
\hline \multicolumn{2}{|c|}{$\mathbf{M}_{e 1}$} & \multicolumn{2}{c|}{$\mathbf{M}_{e 2}$} & \multicolumn{2}{c|}{$\mathbf{M}_{e 3}$} \\
\hline$M_{\rho}$ & $M_{\theta}$ & $M_{\rho}$ & $M_{\theta}$ & $M_{\rho}$ & $M_{\theta}$ \\
\hline $0.20625 q$ & $0.20625 q$ & $-1.0 M$ & $-1.0 M$ & $0.20625 q-1.0 M$ & $0.20625 q-1.0 M$ \\
\hline $0.19366 q$ & $0.19883 q$ & $-1.0 M$ & $-1.0 M$ & $0.19366 q-1.0 M$ & $0.19883 q-1.0 M$ \\
\hline $0.15469 q$ & $0.17656 q$ & $-1.0 M$ & $-1.0 M$ & $0.15469 q-1.0 M$ & $0.17656 q-1.0 M$ \\
\hline $0.15469 q$ & $0.17656 q$ & $-1.0 M$ & $-1.0 M$ & $0.15469 q-1.0 M$ & $0.17656 q-1.0 M$ \\
\hline $0.09023 q$ & $0.13945 q$ & $-1.0 M$ & $-1.0 M$ & $0.09023 q-1.0 M$ & $0.13945 q-1.0 M$ \\
\hline $0.0 q$ & $0.08750 q$ & $-1.0 M$ & $-1.0 M$ & $0.0 q-1.0 M$ & $0.08750 q-1.0 M$ \\
\hline
\end{tabular}

Table 4. Matrix $[\nabla \varphi]$ structure

\begin{tabular}{|l|l|l|}
\hline $2\left[\Phi_{1}\right] \mathbf{M}_{11,1}$ & & \\
\hline $2\left[\Phi_{2}\right] \mathbf{M}_{11,1}$ & & \\
\hline & $\cdots$ & \\
\hline & $\cdots$ & \\
\hline & & $2\left[\Phi_{1}\right] \mathbf{M}_{k l, 1}$ \\
\hline & & $2\left[\Phi_{2}\right] \mathbf{M}_{k l, 1}$ \\
\hline
\end{tabular}


The third problem aims at verifying the plate stiffness conditions (31) $0 \leq u_{r 1} \leq u_{r 1, \max }$. The residual deflection magnitude $u_{r 1}=0.2985 M_{0} R / \varrho \mathscr{K}$ is obtained from the vector $\mathbf{u}_{r}^{* v=1}=[H] \lambda^{* v=1}=\left[\begin{array}{ll}0.2985 & 0.3204\end{array}\right.$ $\left.\begin{array}{llllll}0.1406 & -0.3502 & 0.1345 & 0.1382 & -0.0084 & 0.2362\end{array}\right]^{T}$.

The stiffness condition is satisfied but one must take into account the possible unloadings of the cross-section. Therefore, the stiffness constraint $(31 \check{Z})$ is changed to the more strict one: $0 \leq u_{r 1, \text { sup }} \leq 0.84$.

The upper bound of the deflection is calculated applying the mathematical model (34)-(35). The bending moments (47) are located on the right side of compatibility equations $\left[B_{\lambda}\right] \bar{\lambda}^{v}=\left[B_{r}\right] \mathbf{M}_{r}^{* v}$. The matrix $\left[B_{\lambda}\right]$ is calculated applying the formula $\left[B_{\lambda}\right]=\left[B_{\theta}\right][\nabla \varphi]$. Table 4 illustrates the structure of the matrix $[\nabla \varphi]$. An optimal solution of the problem (34)-(35) are the vectors: $\lambda^{*}=\left[\begin{array}{llllll}0.1064 & 0.6301 & 0.0 & 0.0 & 0.0 & 0.0\end{array}\right]^{T}$, $u_{r 1, \text { sup }}=0.2987 M_{0} R / \circlearrowright \mathcal{K}$.

The residual displacement bound $\tilde{u}_{r 1, \text { sup }}$ for highly reliable structures is calculated applying the mathematical model (34)-(35), stated for the fictitious plate. The

Table 5. Numerical solution of load optimization problem

\begin{tabular}{|c|c|}
\hline & \\
\hline $\begin{array}{l}\text { a) } 0 \leq q \leq q_{\text {sup }}, 0 \leq M \leq M_{\text {sup }} \\
\text { Plate discrete model: } \\
k=2, m=8\end{array}$ & $\begin{array}{l}u_{r i, \max }=0.84 M_{0} R / \mathscr{C} \\
\text { Optimal solution: } \\
u_{r i}^{*}=0.84 M_{0} R / \mathscr{K}, \\
q_{\text {sup }}^{* \nu}=5.7716 M_{0} R^{-2}, \\
M_{\text {sup }}^{* v}=0.8091 M_{0} .\end{array}$ \\
\hline $\begin{array}{l}\text { b) } 0 \leq q \leq q_{\text {sup }}, 0 \leq F \leq F_{\text {sup }} \\
\text { Plate discrete model: } \\
k=8, m=32\end{array}$ & $\begin{array}{l}\text { Plate point deflection } \\
\text { limitation: } \\
u_{r i, m a x}=3.4 M_{0} R / \propto K \\
\text { Optimal solution: } \\
u_{r i}^{*}=3.09 M_{0} R / \propto, \\
q_{\text {sup }}^{* v}=6.7501 M_{0} R^{-2}, \\
F_{\text {sup }}^{* v}=0.0456 M_{0} .\end{array}$ \\
\hline $\begin{array}{l}\text { c) } 0 \leq q \leq q_{\text {sup }}, 0 \leq F \leq F_{\text {sup }} \\
\text { Plate discrete model: } \\
k=8, m=32\end{array}$ & $\begin{array}{l}\text { Plate point deflection } \\
\text { limitation: } \\
u_{r i, \max }=0.1 M_{0} R / \propto K \\
\text { Optimal solution: } \\
u_{r i}^{*}=0.09 M_{0} R / \odot K, \\
q_{\text {sup }}^{* v}=5.7472 M_{0} R^{-2}, \\
F_{\text {sup }}^{* v}=0.0290 M_{0} .\end{array}$ \\
\hline d) $0 \leq q \leq q_{\text {sup }}, 0 \leq M \leq M_{\text {sup }}$ & 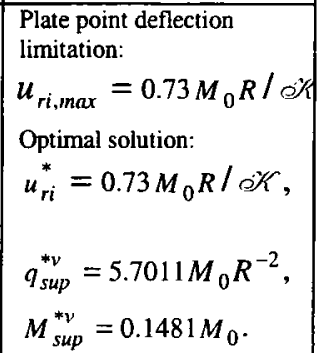 \\
\hline
\end{tabular}

fictitious plate yield constants vector $\tilde{\mathbf{M}}_{0}$ is formulated by following the criterion stating that all nodes yield conditions must be satisfied as equalities. Then the problem (34)-(35) becomes degenerated.

The dissipated energy amount $\widetilde{D}_{\max }=0.7372$ is identified by solving the analysis problem (29)-(31) for the degenerated structure. As the compatibility equations remain the same, the magnitude $\tilde{u}_{r l, s u p}$ is calculated applying the ratio of magnitudes $\bar{D}_{\max }^{r l, \text { sup }}$ and $\widetilde{D}_{\max }$.

Finally, the main problem (29)-(31) optimal solution in terms of the following vectors reads (Table 5):

$q_{\text {sup }}^{* v}=5.7716 M_{0} R^{-2}, M_{\text {sup }}^{* v}=0.8091 M_{0}, \lambda^{*}=[0.1802$

$\left.\begin{array}{lllll}1.8157 & 0.2990 & 0.0 & 0.0 & 0.0\end{array}\right]^{T}, \mathbf{u}_{r}^{*}=\left[\begin{array}{lll}0.8403 & 0.9000\end{array}\right.$

$\left.\begin{array}{lllllll}0.4481 & -1.0843 & 0.4164 & 0.4280 & -0.0260 & -0.7313\end{array}\right]^{T}$.

More numerical applications are presented in Table 5.

\section{Conclusions}

The complementary slackness condition of the mathematical programming theory does not allow to evaluate plate cross-section unloading phenomenon during the shakedown process. Therefore the dual mathematical programming problems cannot be applied directly when determining actual stress and strain fields of plate (the strained state depends upon the loading history). As a result, the load optimization problem at shakedown is to be stated as a couple of problems solved in parallel: the shakedown state analysis problem and the verification of residual deflections bounds.

\section{References}

1. Čyras A. Methods of linear programming for calculations of elastoplastic structures. Leningrad: Strojizdat, 1969. 199 p. (in Russian).

2. Cohn M. Z., Maier G. Engineering plasticity by mathematical programming. Pergamon Press, Elmsford, NY, 1978. $648 \mathrm{p}$.

3. Borkowski A., Kleiber M. On numerical approach to shakedown analysis of structures. Comput. Methods Appl. Mech. Eng., No 22, 1980, p. 101-119.

4. König J. A, Maier G. Shakedown analysis of elastoplastic structures: a review of recent developments. J. Nuclear Engineering Design, Vol. 66, 1981, p. 81-95.

5. Čyras A. Mathematical models for the analysis and optimization of elastoplastic structures. New York: John Wiley, 1983. $121 \mathrm{p}$.

6. Borkowski A., Atkočiūnas J. Optimal Design for Cyclic Loading. IUTAM, Optimization in structural design, Symposium Warsaw/Poland, August 21-24, 1973. SpringerVerlag, 1975, p. 432-440.

7. König J. A. Shakedown of Elastic-Plastic Structures. Amsterdam, 1987. 214 p.

8. Gutkowski W., Bauer J., Iwanow Z. Explicit Formulation of Kuhn-Tucker Necessary Conditions in Structural Opti- 
mization. Comp. \& Structures, Vol. 37, No 5, 1990, p. 753758.

9. Stein E., Zhang G. and Mahnken. Shakedown analysis for perfectly plastic and kinematic hardening materials. Progress in Computational Analysis of Inelastic Structures (Edited by E.Stein), Springer-Verlag, Wien, 1993, p. 175244.

10. Weichert D., Hachemi A., Schwabe F. Application of shakedown analysis to the plastic design of composites. Archive of Applied Mechanics, No 69, 1999, p. 623-633.

11. Giambanco F., Palizzolo L. and Polizzotto C. Optimal shakedown design of circular plates. Journal of Engineering Mechanics, Vol 120, No 12, 1994, p. 2535-2555.

12. Atkočiūnas J. Mathematical Models of Optimization Problems at Shakedown. Mechanics Research Communications, Vol 26, No 3, 1999, p. 319-326.

13. Bazaraa M. S., Shetty C. M. Nonlinear Programming Theory and Algorithms. New York, Chichester, Brisbane, Toronto: John Wiley, 1979. 283 p.

14. Atkočiūnas J. Compatibility equations of strains for degenerate shakedown problems. Computer \& Structures, Vol 63, No 2, 1997, p. 277-282.

15. Chraptovič E., Atkočiūnas J. Mathematical programming applications peculiarities in shakedown problem. Statyba (Civil Engineering), Vol VII, No 2, Vilnius: Technika, 2001, p. 106-114 (in Russian).

16. Čižas A., Čyras A. Analysis of an elastic-plastic structural design considering limited deformations. Lith. Proc. in Mech., No 1, 1967, p. 102-114 (in Russian).

17. Ponter A. R. S. An upper bound on the small displacements of elastic, perfectly plastic structures. J. Appl. Mech., No 39, 1972, p. 959-963.
18. Mróz Z., Weichert D., Dorosz St. (eds.). Inelastic Behaviour of Structures under Variable Loads. Kluwer Academic Publishers, 1995. $523 \mathrm{p}$.

19. Gokhfeld D. A., Chemiavsky O. F. Limit analysis of Structures at Thermal Cycling. Sijthoff \& Noordhoff, 1980. $537 \mathrm{p}$.

20. Atkočiūnas J., Borkowski A., König J. A., Improved bounds for displacements at shakedown. Comput. Methods Appl. Mech. Eng., No 28, 1981, p. 365-376.

21. Ikrin V. A. The prognosis of possible states of structures adapting to unregulated static actions. Irkutsk, Irkutsk University Publishers, 1986. 160 p. (in Russian).

22. Lange-Hansen P. Comparative Study of Upper Bound Methods for the Calculation of Residual Deformations After Shakedown. Denmark, Lygby, 1998. 75 p.

23. T. Belytschko, W. K. Liu. Nonlinear Finite Elements for Continua and Structures. John Wiley \& Sons Ltd., 2000. $300 \mathrm{p}$.

24. Kalanta $S$. The equilibrium finite elements in computation of elastic structures. Statyba (Civil Engineering), No 1, Vilnius: Technika, 1995, p. 25-47 (in Russian).

25. Daniūnas D. Equilibrium finite element method in analysis problems of elastic-plastic plates. Lithuanian Proceedings in Mechanics, No 27, Vilnius, 1984, p. 28-39 (in Russian).

26. Kalanta S. New formulations of optimization problems of elasto-plastic bar structures under displacement constraints. Mechanika, No 5 (20). Kaunas: Technologija, 1999, p. 9 16 (in Russian). 